Research Article in Physics

\title{
Energy harvesting through a piezoelectric sensor
}

\author{
Moojin $\operatorname{Kim}^{1, *}$ \\ ${ }^{1}$ Korean Minjok Leadership Academy, Hoengseong, Gangwon 25268, South Korea \\ *Correspondence: danmuji354@gmail.com
}

(Received: 10/25/2001; Accepted: 12/07/2021; Published: 12/20/2021)

DOI: https://doi.org/10.37906/isteamc.2021.11

\begin{abstract}
Energy harvesting through motion caused by wind is a unique way of finding an alternative energy source for several electronic devices. Piezo-electronic sensors, which harvest energy from small vibrations and movements, are investigated by many researchers nowadays. This paper conducted an experimental study to find an alternative energy source for diverse electronics with forced oscillations from a fan. The relations between the force applied by wind and the oscillation of a paper strip were studied.
\end{abstract}

Keywords: Energy harvesting; Piezoelectric sensor; Wind energy

\section{Introduction}

The concept of energy harvesting is a significant trend, and methods to extract energy are being developed in real-time. Since electronic devices have a specific limit in secondary energy sources, such as pocket batteries, many can benefit from diverse energy harvesting methods. With the help of wind, it is possible to harvest energy from naturally occurring oscillations in electronic systems. One example is energy harvesting with flutter, a self-excited oscillation from a one-sided force, which takes advantage of the self-excitation of an object caused by wind. In this paper, piezoelectric sensors will use energy harvesting with oscillations induced by sinusoidal force from the wind.

Energy harvesting with piezoelectric sensors has several advantages over electromagnetic sensors. They are lightweight, have high sensitivity, and have simple structures. This paper analyzes a model with a piezoelectric sensor attached to a paper strip by analyzing voltage change as wind speed was varied with a fixed frequency.

\section{Research Design, Data collection, and analysis Methods}

2.1. Study 1: Investigation of the linearity between the current and wind speed

As an attempt to harvest wind energy, mainly three parts were used: a fan, a paper strip, and a piezoelectric sensor. As shown in figure 1, the fan was at a $15 \mathrm{~cm}$ distance from the paper strip, of 0.5gram weight and size of $162 \times 24 \times 0.11 \mathrm{~mm}$. At $7 \mathrm{~mm}$ from the edge of the paper strip, an LDT0-028K piezoelectric sensor with negligible mass was attached. The fan was set to rotate, which led to a sinusoidal force. Then to set the force on the system as a variable, the wind speed was set to three levels, each of $1.3 \mathrm{~m} / \mathrm{s}, 1.7 \mathrm{~m} / \mathrm{s}$, and $2.0 \mathrm{~m} / \mathrm{s}$. 


\subsubsection{Design of Study 1}

Since this system is a forced oscillation, the model of the forced oscillation can be obtained as

$$
\mathrm{m} \ddot{x}+k x+c \dot{x}=F \cos (\omega t)=\mathrm{F} e^{i \omega t}
$$

where $\omega$ represents the angular velocity of the oscillation, m $\ddot{x}$ mass times acceleration, $k x$ the returning force, and $c \dot{x}$ the damping force (15.7: Forced Oscillations, 2020). In order to find the amplitude of this oscillation, the following steps are taken:

$$
\mathrm{m} \ddot{x}+k x+c \dot{x}=F \cos (\omega t)=\mathrm{F} e^{i \omega t}
$$

can be transformed into

$$
\ddot{X}+\frac{c}{m} \dot{X}+\omega_{0}^{2} X=\frac{F}{m} e^{i \omega t}
$$

We can derive a function as a solution for the amplitude as

$$
X=X_{0} e^{i(\omega t-\delta)},
$$

where $\delta$ is the phase difference between the driving force and the natural frequency of the unforced system. By obtaining the solution of the equation, we finally obtain the amplitude of the oscillation, as shown below:

$$
X_{0}=\frac{\frac{F}{m}}{\sqrt{\left(\omega_{0}^{2}-\omega^{2}\right)^{2}+\left(\omega \frac{c}{m}\right)^{2}}} .
$$

With the equation for the amplitude derived, we now know that only the force applied on the paper strip and the frequency are the variables for the oscillation. However, in this specific study, the frequency of the driving force was set constant. Therefore, the experiment with the change of the level of wind from one to three is able to show the transformation of the amplitude of the oscillation at a fixed frequency. The oscillation amplitude is a variable for the function of output voltage, or the voltage harvested, leading to the function of output voltage as a function of force. Since the force applied to the system is also a function of the wind velocity of the fan, the output voltage can finally be modeled as

$$
V_{\text {out }}=k v^{n}
$$

where $v$ is the air velocity and $\mathrm{n}$ determines the exponent of the power-law.

\subsubsection{Data collection and analysis methods for Study 1}

The output voltage was measured through the three wind levels and was analyzed through the chisquared method. 


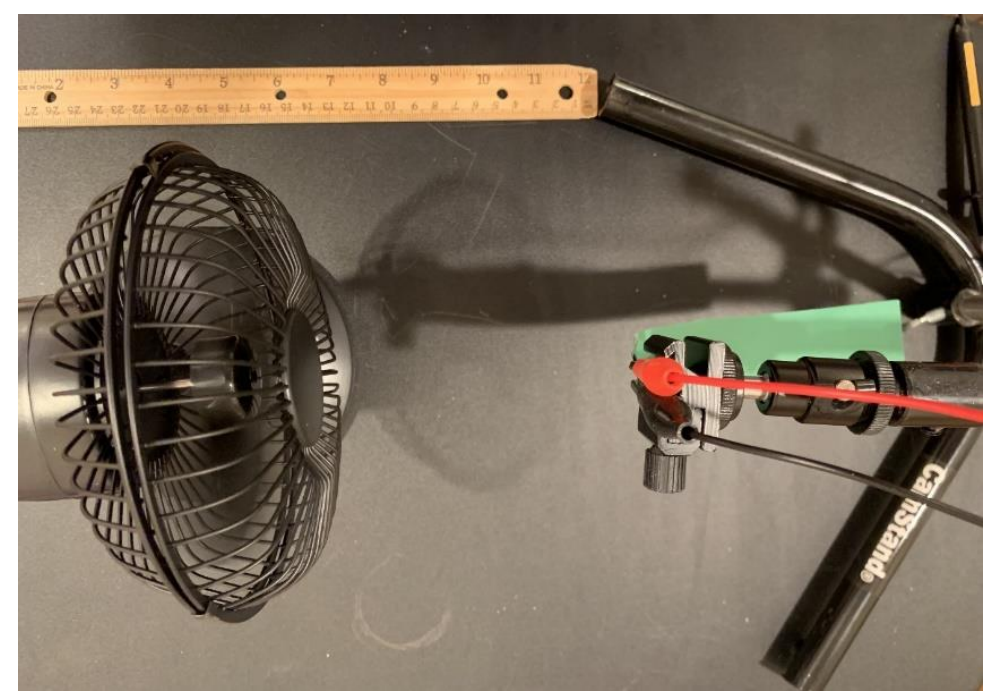

Figure 1. The experimental setup

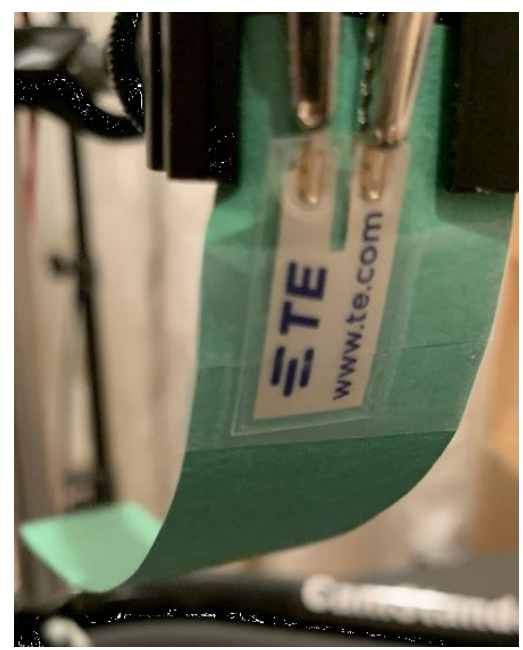

Figure 2. The LDT0-028K piezoelectric sensor

\section{Results}

\subsection{Results of Study 1}

To collect the data of the energy generated by the oscillation of the paper strip, a piezoelectric sensor LDT0-028K was used. Energy harvesting was done through three levels of wind, each of $1.3 \mathrm{~m} / \mathrm{s}, 1.7 \mathrm{~m} / \mathrm{s}$, and $2.0 \mathrm{~m} / \mathrm{s}$, and the voltage increased through the increase of wind speed. As shown in figure 3 , as the wind speed increases, the voltage fluctuates around larger values. The data shows a sinusoidal shape due to the fan's movement, which was applied similarly for the three data sets. 

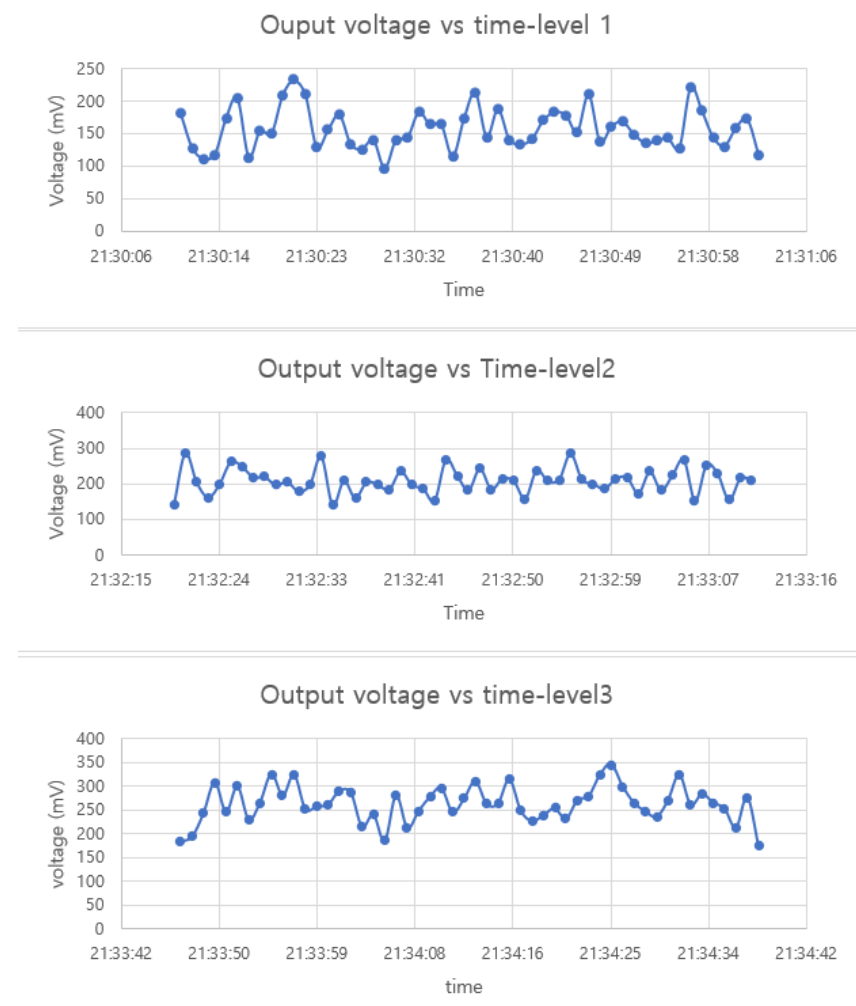

Figure 3. Comparison of the output voltage of 3 different wind speeds

\subsubsection{Linearity between wind speed and output voltage}

The data were fit to a power-law model using Chi-squared analysis. Taking the function $V_{\text {out }}=k v^{n}$ and converting it to a linear model by taking natural $\log$ arithms of both sides, we obtain $\log V_{\text {out }}=\log k+$ $n \log v$, making the Chi-squared analysis simple. By setting $\mathrm{x}$ as the natural logarithms of air velocity, while setting $y$ as the natural logarithms of the output voltages, a Chi-squared analysis with the six voltage values for each level was done to find the best fit for each parameter. A best is the best slope for $x$ and $\mathrm{y}$, and $\mathrm{B}$ best is $\log k$. The chi-square minimum was 1.87 , with a good fit range of 0.55 to 5.45 . With the constants for the A best and B best, the exponent of the power law was 2.56 and the constant $\mathrm{k}$ was found, and the constant $\mathrm{k}$ was found.
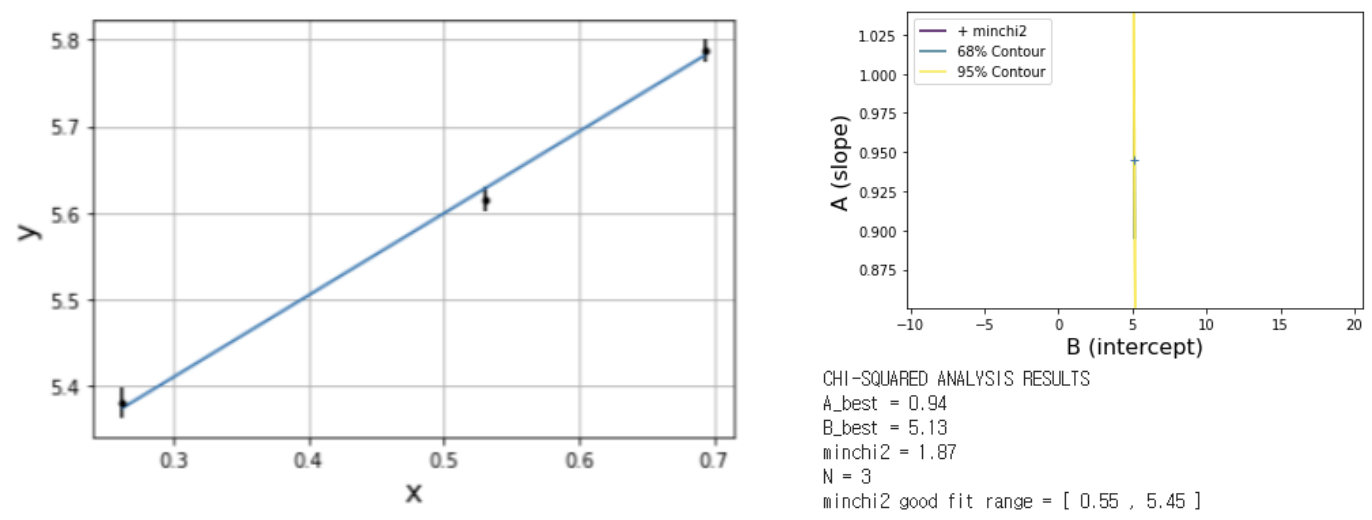

Figure 4. Result of Chi-square analysis 


\section{Discussion and Synthesis}

The wind-driven oscillations have demonstrated the conversion of wind energy into electrical energy. In this paper, a model of forced oscillation has been set up to study the produced voltage through the oscillation of the paper strip with the piezoelectric sensor attached. The output voltage with the same wind frequency in different wind speeds showed a power law increase as the wind speed was controlled through three levels. The final equation derived through the results matched the given data. The wind harvester, through a piezoelectric pickup, was able to harvest electrical energy. However, the frequency may not be constant in natural settings; a further investigation would be needed to understand the effects of this extra variable.

Acknowledgments: I would like to express my deep gratitude to Dr.Witkov, my research supervisor, for his infinite attention and helpful advice. I would like to thank Gabe for his guidance and assistance in keeping my work in control.

\section{References:}

Libretexts. (2020, November 5). 15.7: Forced oscillations. Physics LibreTexts. Retrieved October 1, 2021, from https://phys.libretexts.org/Bookshelves/University_Physics/Book\%3A_University_Physics_(Open Stax)/Book\%3A_University_Physics_I__Mechanics_Sound_Oscillations_and_Waves_(OpenStax)/15\%3A_Oscillations/15.07\%3A_Forced _Oscillations. 